(1) Is there a role for EUS as one of the initial imaging modalities? (2) Can EUS replace other diagnostic procedures, especially ERCP? (3) In which period of the acute attack may EUS and EUSFNA play a role?

The published studies about these issues are quite limited. They mainly focused on the role of EUS in cause finding. A potential issue which has not really been addressed is the role of EUS in the early management of complications of an acute pancreatitis such as pancreatic fluid collections and infection.

The definition of an acute attack especially with regard to the exact time frame has not clearly been defined yet. Clinically the acute attack is present as long as the pancreatic enzymes are elevated. However, from our experience even though the enzymes have returned to normal values, the pancreas parenchyma can be still edematous and fluid collections may be present. EUS may provide information about the status of the parenchymatous stage of inflammation in addition to laboratory findings. This information can be important for further management of patient and predicting the further progress of the attack.

CT has been the gold standard imaging to access the severity of acute pancreatitis using the Balthazar computed tomography index which grades the severity based on pancreatic inflammation and necrosis. There are no published studies comparing EUS and $\mathrm{CT}$ in grading the severity of the disease. EUS provides good locoregional visualization, however extension of the necrosis is still best visualized by CT. Therefore EUS cannot replace CT as the index imaging. The situation is supposed to be different with regards to cause finding.

\title{
Studies on yield of EUS
}

Frossard et. al. managed to determine the cause of acute pancreatitis in $77 \%$ of 168 patients by EUS. They misdiagnosed idiopathic pancreatitis as having biliary stones in 5 patients and IPMT in 1 patient [1].

\section{Acute pancreatitis: The acute attack. Acute recurrent pancreatitis}

S. Seewald, S. Omar, N. Soehendra

Dept. of Interdiscipl. Endoscopy, Univ. Hospital Hamburg-Eppendorf

Due to the well accessibility of the pancreatobilary system, the potential role of EUS and EUS-FNA in an acute attack of a first episode or recurrent idiopathic pancreatitis seems to be an issue. Since EUS is competing with other imaging modalities such as CT, MRCP and ERCP, the following questions have to be raised.

Correspondence: Dr. med. Stefan Seewald · Dept. of Interdiscipl. Endoscopy Univ. Hospital Hamburg-Eppendorf · Martinistr. 52 - 20246 Hamburg · Phone:

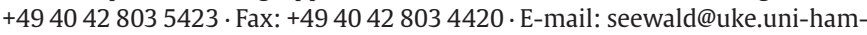
burg.de

Bibliography: Endoscopy 2006; 38 (S1): S21-S22 @ Georg Thieme Verlag KG Stuttgart · New York · ISSN 0013-726X · DOI 10.1055/s-2006-946646

In a study of Tandon et al. with 31 patients, EUS demonstrated an etiology in two-thirds of patients with idiopathic acute pancreatitis. All these patients had undergone transabdominal ultrasound and CT scan before EUS. Especially in microlithiasis, pancreatic cancer and chronic pancreatitis, EUS was very accurate. The missed diagnoses were pancreatic duct stones, pancreas divisum, intraductal papillary mucinous tumor and sphincter of Oddi dysfunction [2]. A criticism of this paper was that patients with significant consumption of alcohol were included into this study together with patients with first attack of acute pancreatitis, who will usually not have any further attacks $[3,4]$.

However, in a prospective study of EUS in primary vs. recurrent idiopathic acute pancreatitis by Yusoff et al., the yield of EUS in primary acute pancreatitis was not significantly different from recurrent idiopathic pancreatitis. Therefore it was reasonable to perform EUS after the first attack. In this study the overall yield of positive finding was $29.2 \%$. The lower rate compared to other studies can be explained by the fact that authors excluded pa- 
tients with significant alcohol consumption. Furthermore, chronic pancreatitis noted by EUS were not included as a positive finding [5].

A study by Coyle et al. determined the diagnostic utility of ERCP with sphincter of Oddi manometry, bile analysis and EUS in patients with acute and acute recurrent idiopathic pancreatitis [6]. Authors concluded that EUS was useful in detecting biliary disease and anatomic anomalities and valuable in detecting early changes of chronic pancreatitis. EUS identified all the tumors. In this study, $31 \%$ of the patients had sphincter Oddi dysfunction, which logically cannot be diagnosed by EUS.

\section{Can EUS preclude ERCP}

A clinically important question is if EUS is able to avoid inappropriate cannulation of the papilla. In the management of patients with suspected biliary pancreatitis, the role of emergency ERC is still controversial and EUS theoretically could help to restrict ERC to patients with documented stones or sludge in the biliary tract.

In a study of Prat et al. the value of early EUS of the bile duct before endoscopic sphincterotomy for acute biliary pancreatitis was investigated. One hundred twenty three patients with suspected biliary pancreatitis underwent EUS followed by selective endoscopic sphincterotomy during the same procedure if choledocholithiasis was found. Bile duct stones were found in $27 \%$ of the patients. The proportion of patients with stones was higher when EUS was performed within 3 days after the onset of symptoms as compared with EUS performed after 3 days (33\% vs. $18 \%$, $\mathrm{p}<0.05)$. ERC with endoscopic sphincterotomy was performed immediately after EUS in the same endoscopic session. There was no false positive EUS result and no complication directly related to EUS. Only in 3 patients (2.4\%) EUS imaging of the bile duct was unsatisfactory. None of the patients with severe pancreatitis and negative EUS had recurrence of biliary symptoms during follow up [7].

In a prospective study by Napoleon et al. the negative predictive value of EUS for the diagnosis of CBD stones was 95.4\%. Authors clearly demonstrated that normal findings at EUS obviated the need for ERC in the great majority of the patients with suspicion of CBD stones [8].

A promising but still experimental new approach has recently been presented by Rocca et al. in the treatment of CBD stones. EUS and ERCP were performed with a single echoendoscope. The strategy is called endoscopic ultrasonography retrograde cholangiopancreatography (EURCP). The authors used an Olympus fiberoptic oblique-viewing mechanical radial echoendoscope, with an operating channel of $2.2 \mathrm{~mm}$ and a standard elevator with a single frequency of $7.5 \mathrm{MHz}$. This combination procedure took an average time of 27 minutes. Bile duct cannulation was successful in 15 of 16 cases (94\%). With technological improvements EURCP might have a role as first line management for patients with suspected biliary pancreatitis [9].

\section{EUS in the management of early complication}

EUS may play a potential role in the management of early complication of acute pancreatitis such as acute fluid collections and infection. Due to the good accessibility of EUS, infected necrosis or fluid collections could be punctured and drained. However, in this early stage, the necrosis and acute fluid collections are more diffuse and not encapsulated, so an effective irrigation is almost impossible. The only possible role of EUS-FNA is to confirm the presence of infection if clinically indicated. As far as we know there is no study comparing EUS-FNA with CT guided aspiration.

\section{Conclusion}

EUS can be an initial imaging modality in the first attack or recurrent idiopathic pancreatitis for cause finding, especially if patients were suspected to have biliary etiology. There is a role of EUS in preventing unnecessary ERC. EUS guided transgastric FNA may be performed to confirm infection. EUS guided transgastric drainage of necrosis or fluid collections do not play a therapeutic role in the acute attack.

\section{References}

${ }^{1}$ Frossar JL, Sosa-Valencia L, Amouyal G et al. Usefulness of endoscopic ultrasonography in patients with "idiopathic" acute pancreatitis. Am J Med 2000; 109: 96-200

${ }^{2}$ Tandon M, Topazian M. Endoscopic ultrasound in idiopathic acute pancreatitis. Am J Gastroenterol 2001; 96: 705-709

3 Chen RY, Hawes RH. Idopathic acute pancreatitis: Is EUS worth doing? Am J Gastroenterol 2002; 97: 1244-1246

${ }^{4}$ Ballinger AB, Barnes E, Alstead EM et al. Is intervention necessary after a first episode of acute idiopathic pancreatitis? Gut 1996; 38: 293 295

${ }^{5}$ Yusoff IF, Raymond G, Sahai AV. A prospective comparison of the yield of EUS in primary vs. recurrent idiopathic acute pancreatitis. Gastrointest Endosc 2004; 60: 673-678

${ }^{6}$ Coyle WJ, Pineau BC, Tarnasky PR et al. Evaluation of unexplained acute and acute recurrent pancreatitis using endoscopic retrograde cholangiopancreatography, sphincter of Oddi manometry and endoscopic ultrasound. Endoscopy 2002; 34: 617-623

${ }^{7}$ Prat F, Edery J, Meduri B et al. Early EUS of the bile duct before endoscopic sphincterotomy for acute biliary pancreatitis. Gastrointest Endosc 2001; 54: 724-729

${ }^{8}$ Napoleon B, Dumortier J, Keriven-Souquet O et al. Do normal findings at biliary endoscopic ultrasonography obviate the need for endoscopic retrograde cholangiography in patients with suspicion of common bile duct stone? A prospective follow-up study of 238 patients. Endoscopy 2003; 35: $411-415$

${ }^{9}$ Rocca R, De Angelis C, Castellino F et al. EUS diagnosis and simultaneous endoscopic retrograde cholangiography treatment of common bile duct stones by using an oblique-viewing echoendoscope. Gastrointest Endosc 2006; 63: 479-484 